\title{
Pandemic publication: correction and erratum in COVID-19 publications
}

\author{
Shima Moradi ${ }^{1}$ (D) $\cdot$ Sajedeh Abdi $^{1}$
}

Received: 21 September 2020 / Published online: 21 November 2020

(c) Akadémiai Kiadó, Budapest, Hungary 2020

\begin{abstract}
This commentary identifies and characterizes correction and erratum in COVID-19 publications with a scientometric approach by considering their rate of growth, reasons for correction, the time-span between publishing the original and corrected versions, as well as their citation status in four questions. It also suggestions to solve the current issues regarding indexing, retrieving, publishing, and research evaluation.
\end{abstract}

Keywords Correction $\cdot$ Erratum $\cdot$ Indexing $\cdot$ Pandemic $\cdot$ Papers

\section{Introduction}

Articles are essential forms of scholarly communication in the health sciences. In the past, only the printed journals were available. By the end of the twentieth century, the rate of online publication has increased (Vervaart 2014), eliminating surplus printing costs, improving journal accessibility, and speeding up the publishing procedure. To maintain the quality, journals examine manuscripts by peer-reviewing to meet particular criteria according to the scope, methodology, and scientific rigor. The publishing procedure is time-consuming; therefore, academia has raised new scholarly approaches like open peer-reviewing (Pöschl 2012; Walker et al. 2015), preprinting (Johansson et al. 2018; Ross-Hellauer et al. 2017), post-publication peer review (Tennant 2018), and repositories to improve the process and broader scholarly research ecosystem.

The increasing volume of publications may cause potential errors (Molckovsky et al. 2011). Some are examples of scientific misconduct, which lead to retraction and others contain typographical errors (Cox, Cox, and Cox, 2017) which are neglectable. Last but not least, some errors may occur unintentionally in any phase of research such as authors' affiliations (King 2001), mislabeling of figures, incorrect numbers, or references. The correction version, also known as Erratum or Corrigendum (Erfanmanesh and Morovati

Shima Moradi

moradi@nrisp.ac.ir

Sajedeh Abdi

sajedeh.abdi@shahed.ac.ir

1 Policy Evaluation, and Science, Technology and Innovation Monitoring Department, National Research Institute for Science Policy (NRISP), Teharn, Iran 
Table 1 Distribution OF ARTICLE corrections DuRING COVID-19 pandemic

\begin{tabular}{llcl}
\hline Time & Article & Correction & Retraction \\
\hline Jan-20 & 10,938 & 25 & 0 \\
Feb-20 & 1414 & 1 & 2 \\
Mar-20 & 4252 & 8 & 0 \\
Apr-20 & 10,826 & 27 & 1 \\
May-20 & 16,186 & 43 & 0 \\
Jun-20 & 20,522 & 45 & 0 \\
Jul-20 & 25,388 & 69 & 0 \\
Aug-20 & 4804 & 18 & 0 \\
Sep-20 & 1334 & 3 & 0 \\
Oct-20 & 683 & 0 & 0 \\
Nov-20 & 446 & 2 & 0 \\
Dec-20 & 1411 & 18 & 0 \\
SUM & 98,204 & 259 & 3 \\
\hline
\end{tabular}

2019), briefly explains the errors of an upcoming, under review, or published article and points to the correct form. With respect to science ethics, the authors and editors have to correct any errors in their publication as soon as they notice it via a clear correction notice (Scarlett 2017). The Committee on Publication Ethics ${ }^{1}$ and reputable publishers like Emerald $^{2}$ provide policies and guidelines for such conditions. Publishing inaccurate data and information in some cases may distract science from the right track, especially in health sciences.

According to the COVID-19 pandemic, the related publication rate has grown dramatically, as well as misinformation (Pennycook et al. 2020), leading to potential challenges regarding dissemination speed, scholarly communication, and data availability in the early stages of the epidemic. The scientific community can apply the findings of these researches more effectively to determine the right foundations and discover an efficient solution to this pandemic (Homolak et al. 2020). This commentary is concerned with rising corrections and suggestions in this respect. We believe this is the approach we can take to contribute to this global challenge using a scientific attitude.

At the time of preparing this commentary on Aug 6, 2020, the Dimensions database ${ }^{3}$ has indexed 98,204 records on COVID-19, which reflects the scholars' attempt to find a cure for this pandemic. These records included 20,849 preprints and 77,355 items were in the form of Article, Book, Book Chapter, Monograph, Proceedings, Corrections, and Retractions (the two latter forms are demonstrated in Table 1). The first relevant researches were published in January 2020, known as the COVID time. This commentary identifies and characterizes correction and erratum in COVID-19 publications with a scientometric approach and considers the growth rate of publications, reasons for correction, the interval between publishing the original and correction versions, and their citation status in four questions as shown in below Table.

\footnotetext{
1 https://publicationethics.org/files/retraction-guidelines.pdf.

2 https://www.emeraldgrouppublishing.com/services/authors/author-policies/article-withdrawal-correction.

3 https://docs.google.com/spreadsheets/d/1-kTZJZ1GAhJ2m4GAIhw1ZdlgO46JpvX0ZQa232VWRmw/ edit\#gid=2034285255 [Accessed August 6, 2020].
} 


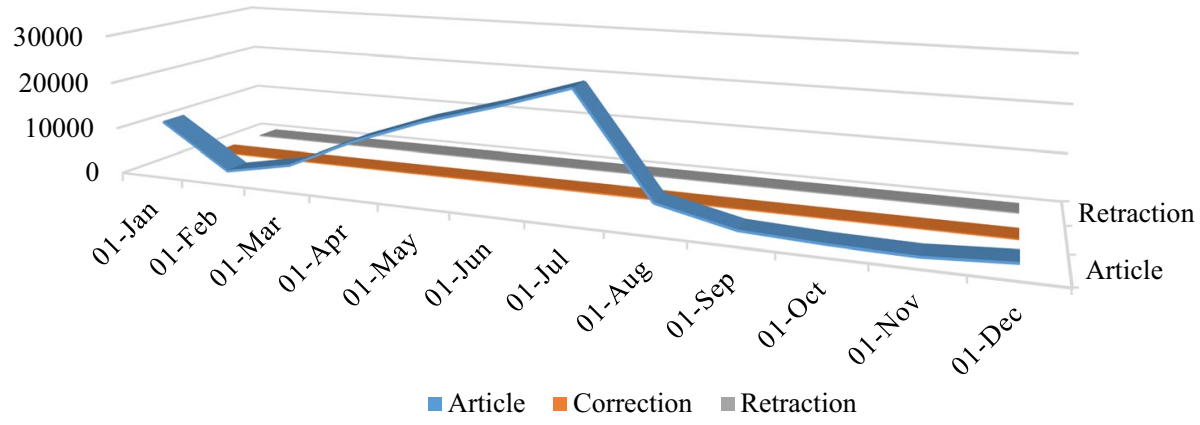

Fig. 1 Distribution of article corrections during COVID-19 pandemic

\section{The growth rate of COVID-19 publications}

Considering the current emergency status, it seems that there has been a rush in conducting researches (Soltani and Patini 2020), peer-reviewing, publishing, and sharing the latest findings, which may be the reason for three retractions and 259 corrections so far. Figure 1 and Table 1 displayed the highest rate of growth in corrections during April (27 records), May (43 records), June (45 records), and July (69), with 10,826, 16,186, 20,522, 25,388 records, respectively. It seems that the high growth of publications can lead to increasing correction rates. However, for a better understanding of this relationship, it is suggested to study it for a more extended period.

Moreover, the publishers have provided the revised versions with different titles like Correction (167 records), Erratum (40 records), Corrigendum (34 records), and Addendum (9 records) according to retrieving perspective. The error in research may happen anytime; however, authors should consider even minor mistakes and eliminate them by informing the publishers. Furthermore, it is essential to provide the respective amendments as soon as possible to avoid any possible misusage.

\section{The reasons for corrections of COVID-19 publications}

The Correction versions were retrieved using google scholar to identify the correction notices as well as reasons for revisions. There are several explanations for publication corrections that could occur in any section of an article. For COVID-19 publications, the corrections have been related to authors' information ( 76 records), tables ( 29 records), results and findings (28 records), introduction ( 25 records), figures ( 25 records), and unknown (17 records). It is worth mentioning that some articles contained up to four different errors that mostly addressed publishers (correction of an earlier incomplete version, article web address, or attaching a statement), which is demonstrated in Table 2.

According toTable 2, there were a number of records with an unknown reason for corrections, ${ }^{4}$ it appeared as if the publishers had no obligation to clarify the correction type. Therefore, if a correction version is a solution to resolve misinformation or fault in the

${ }^{4}$ Sample: Wei Su et al. (2020). 
Table 2 Reasons for correction upon sections of articles

\begin{tabular}{lcl}
\hline Section & Number & Frequency (\%) \\
\hline Authors' Information & 76 & 24.9 \\
Table & 29 & 9.4 \\
Results & 28 & 9.1 \\
Introduction & 25 & 8.1 \\
Figures & 25 & 8.1 \\
Title & 14 & 4.5 \\
Acknowledgment & 12 & 3.9 \\
References & 12 & 3.9 \\
Discussion, Conclusion & 10 & 3.2 \\
Appendix & 8 & 2.6 \\
Note, Footnote & 5 & 1.7 \\
Methodology & 9 & 3 \\
Abstract & 6 & 2.2 \\
Keywords & 1 & 0.4 \\
Others & 29 & 9.4 \\
Unknown & 17 & 5.6 \\
Total & 306 & 100 \\
\hline
\end{tabular}

publication process, the message has to be stated clearly by addressing the corrections in the article. Solving this problem requires a specific uniform framework among all publishers since the problem has been observed in many publishers.

It should be noted that providing the correct unique forms for the Authors is important from indexing and research evaluation perspectives. Several forms in name and affiliation do not reflect the real impact of an article in the scientific society, and the author may remain anonymous. Furthermore, for those with the same name and little knowledge of scientometrics indicators, this can create a situation to promote scholarly impact by registering other scholars' articles in their resume to manipulate their $\mathrm{H}$ index or article numbers (Abdi 2019). As for COVID-19 publications, there were articles with the wrong author name (Marco Hedemperger instead of Marco Heidempergher) ${ }^{5}$ affecting both evaluation procedures and databases like Web of Science or Scopus in gathering author profiles and analyzing scholarly impact through known indicators.

Besides, a misconception of finding or misusage could occur when providing incorrect information in other sections as the appendix, introduction, conclusion, or title, which is certainly not the goal of any researcher. Especially in the COVID-19 pandemic, some researches ${ }^{6}$ may lead to patenting or medicine production, so accuracy is of high importance. On the other hand, accurate information in references or acknowledgment increases the validity of the research.

\footnotetext{
5 Rombolà et al. (2020).

${ }^{6}$ Like: https://www.usatoday.com/story/news/health/2020/06/04/coronavirus-journal-spikes-study-raisingconcerns-covid-19-drug/3146897001/.
} 


\section{The publishing time-span between the original and corrections in COVID-19 publication}

The findings displayed 0 to $>100$ (up to 178) days as the time-span between publishing the original and correction versions, which was quite variable. Content analysis of correction notices also revealed different procedures of journals toward correction versions that led to mentioning different dates by publishers. Immediate corrections with zero time-span contained minor mistakes like the author's name or affiliation that were easy to discover. In contrast, a major challenge in COVID-19 publication evaluation might arise in errors in Methodology, References, or Acknowledgment sections. Furthermore, a period longer than three months was surprising because these are the only reliable sources for countries to cure and immune their citizens.

Table 3 represented 16 records with unknown titles, ${ }^{7}$ indicating that the journal has not provided a specific date for the initial online publication or correction, which makes it impossible to identify the time-span between different versions of a single article.

\section{Citation rate in COVID-19 corrections}

Regarding our data, 259 corrections had received 163 citations in Dimensions. The samples comparing the two versions were presented below (Figs. 2, 3). The articles "Clinical predictors of mortality due to COVID-19 based on an analysis of data of 150 patients from Wuhan, China" and "A new coronavirus associated with human respiratory disease in China" have received 736 (955 Altmetric scores) and 1406 (1799 Altmetric scores) citations, respectively, while their corrections have respectively received 90 (2 Altmetric scores) and 28 (3 Altmetric scores) citations. The citation analysis of corrections in COVID-19 publications can be a topic for further studies. These figures presented the impact of two versions of the above articles. The high number of citations and altmetric scores as known metrics in scientometric and altmetric as well as the decrease of these numbers for the corrected versions are noticeable. Corrections are necessary to academia; however, they could have a negative impact on the respective research, too. This is only the result of initial observations, and for a definite conclusion, a larger population is required.

There are concerns regarding COVID-19 publications, and the following suggestions are presented in this regard.

\section{Retrieving}

Browsing several databases to gather the most comprehensive dataset of COVID-19, we found misinformation or wrong data in a number of fields. It seems that most databases rush to index more resources for better information dissemination.

\footnotetext{
${ }^{7}$ Sample: Chen et al. (2020).
} 


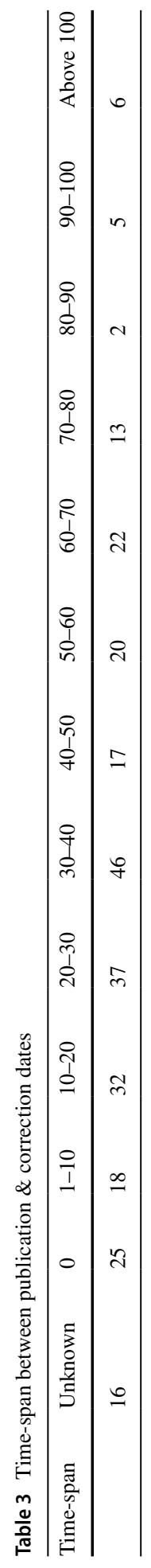


Fig. 2 Citation rate in sample 1

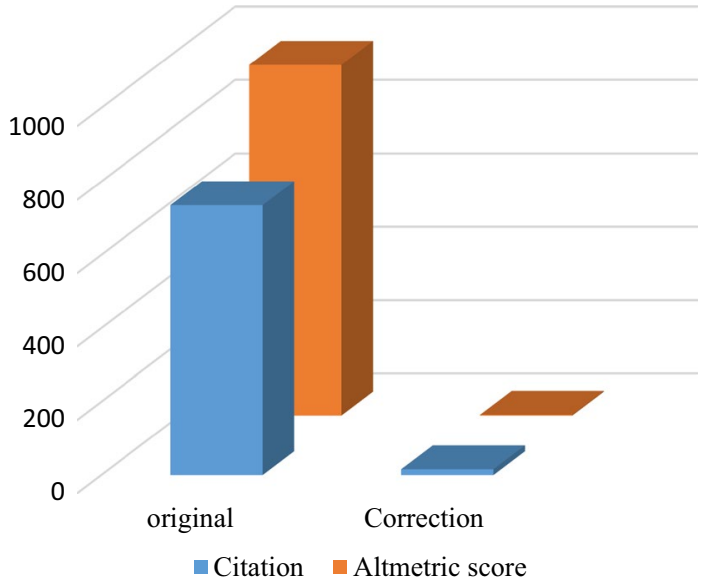

Fig. 3 Citation rate in sample 2

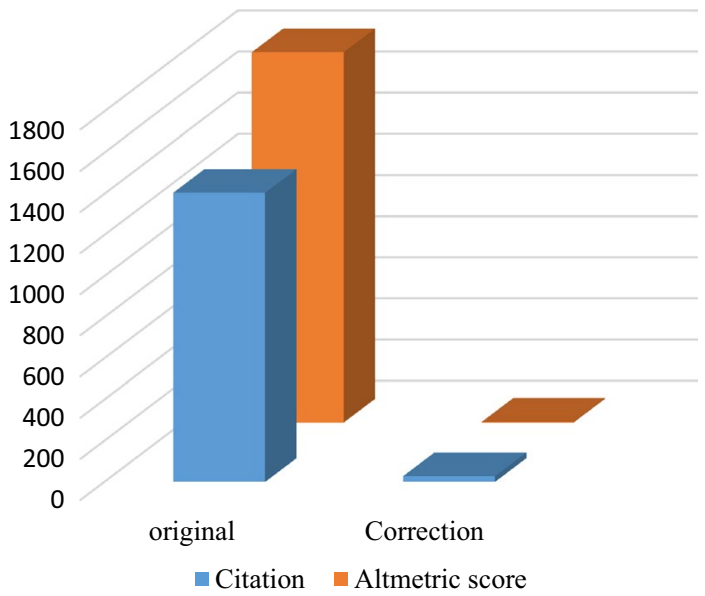

\section{Authorship}

When submitting a manuscript, the author should review all sections, especially those prepared by co-authors, and inform the publisher of error regardless of the manuscript situation, whether it is under peer-review or after the article is published.

\section{Research evaluation}

As for interpreting citations, it is important to consider their nature (positive, negative, and neutral citation) (Bar-Ilan and Halevi 2017), context, and normalization upon the research subject. Moreover, evaluating researches based on citation counts without noticing correction or retracted versions is not enough (Janavi and Moradi 2018). In the meantime, the growth in preprints is a phenomenon observed in COVID-19 publications (Majumder and Mandl 2020). Preprints are efficient channels for quick responses in scientific communities 
(Zhang et al. (2020); however as the preprints are the final versions of an article with no quality assurance like peer-review, relying on them in this crisis can be a concern for the practitioner and scholars in health science.

As for suggestions, we believe open science is the key to face any emergencies like this one. The best approach can come about with international scientific contribution, availability of data and findings (Belli et al. 2020), and modifying channels and scientific infrastructure as follows:

- Assigning an ID definition for the author that includes personal information, affiliation, number of articles, citations, and so forth to prevent all possible ambiguities. This ID, like DOI or research ID, is applicable when journals mandate providing this ID when submitting a manuscript, so that misspelling or missing in any field is recoverable.

- Databases have to provide the organization's name using documentation tools to avoid possible typographical errors. They can also provide an authentication system to confirm the correction of entry information in the form of electronic mail or SMS and not index records with missing fields.

- Information on the organization is crucial for retrieving. Publishers and database designers are better at allocating a unique label for other formats of an article. Nowadays, the corrections are also indexed as Erratum or Corrigendum, which is confusing.

- Developing a transparent mechanism for correcting publications, correction notices, linkage of the original and correction in the web page of the article, accelerating the publication of corrections, and allocating a specific section for corrections in journal table of contents.

- Suggesting authors to publish the manuscript in the form of preprints and use Publons and Pubpeer to have an initial evaluation before submitting the article.

- Encouraging publishers and authors to publish the corrections in social networks similar to the original format to inform users.

- Reviewing and revising COVID-19 indexed publications in databases by experts.

- Preventing 'COVID opportunism' by revisiting COVID-19 publications, and ensuring that there is no corrections version of the article while citing an article.

- Instructing the researchers on how to evaluate publications and avoid unintentional scientific misconduct.

- Responsible research requires culture-building. Informing publishers about an honest error is an ethical conduct. Correction differs from retraction and guarantees quality in academia.

- Paying attention to the role of librarians as scholarly communication experts in information dissemination and finding corrections (Dixie et al. 2003).

\section{References}

Abdi, S (2019). Identification of the Issues and Organizing Challenges of Authority Control in Bibliometric Databases and Suggestions for Improvement. (Unpublished Master Dissertation).Shahed University, Tehran, Iran.

Bar-Ilan, J., \& Halevi, G. (2017). Post retraction citations in context: A case study. Scientometrics, 113(1), 547-565.

Belli, S., Mugnaini, R., Baltà, J., \& Abadal, E. (2020). Coronavirus mapping in scientific publications: when science advances rapidly and collectively, is access to this knowledge open to society?. Scientometrics 
Chen, X., Liu, Y., Gong, Y., Guo, X., Zuo, M., Li, J., \& Huang, Y. (2020). Perioperative management of patients infected with the novel coronavirus: recommendation from the Joint Task Force of the Chinese Society of Anesthesiology and the Chinese Association of Anesthesiologists. Anesthesiology, 132(6), 1307-1316.

Cox, D., Cox, J. G., \& Cox, A. D. (2017). To Err is human? How typographical and orthographical errors affect perceptions of online reviewers. Computers in Human Behavior, 75, 245-253.

Erfanmanesh, M., \& Morovati, M. (2019). Published errors and errata in library and information science journals. Collection and Curation, 38(3), 61-67.

Homolak, J., Kodvanj, I., \& Virag, D. (2020). Preliminary analysis of COVID-19 academic information patterns: A call for open science in the times of closed borders. Scientometrics, 124(3), 2687-2701.

Janavi, E., \& Moradi, S. (2018). Citation Fate of World Retracted Articles: The comparative study of humanities, medical science, engineering science, and pure science. Iranian Journal of Information Management, 4(1), 25-40.

Johansson, M. A., Reich, N. G., Meyers, L. A., \& Lipsitch, M. (2018). Preprints: An underutilized mechanism to accelerate outbreak science. PLoS medicine, 15(4), e1002549.

Jones, D. A., Watson, M. M., Comegys, M., Burnett, A., \& Tucker, B. (2003). Errata and retractions in electronic journals. Journal of Hospital Librarianship, 3(2), 19-27.

King, C. R. (2001). Ethical issues in writing and publishing. Clinical Journal of Oncology Nursing, 5(3), 19-23.

Majumder, M. S., \& Mandl, K. D. (2020). Early in the epidemic: impact of preprints on global discourse about COVID-19 transmissibility. The Lancet Global Health, 8(5), e627-e630.

Molckovsky, A., Vickers, M. M., \& Tang, P. A. (2011). Characterization of published errors in high-impact oncology journals. Current Oncology., 18(1), 26-32.

Pennycook, G., McPhetres, J., Zhang, Y., \& Rand, D. (2020). Fighting COVID-19 misinformation on social media: Experimental evidence for a scalable accuracy nudge intervention. Psychological Science, 31(7), 770-780.

Pöschl, U. (2012). Multi-stage open peer review: Scientific evaluation integrating the strengths of traditional peer review with the virtues of transparency and self-regulation. Frontiers in computational neuroscience, 6, 33.

Rombolà, G., Heidempergher, M., Pedrini, L., et al. (2020). Correction to: Practical indications for the prevention and management of SARS-CoV-2 in ambulatory dialysis patients: Lessons from the first phase of the epidemics in Lombardy. J Nephrol, 33, 197. https://doi.org/10.1007/s40620-020-00729-w.

Ross-Hellauer, T., Deppe, A., \& Schmidt, B. (2017). Survey on open peer review: Attitudes and experience amongst editors, authors and reviewers. PLOS ONE, 12(12), e0189311.

Scarlat, M. M. (2017). Erratum, corrigenda etemendatio or mistake, correction and amendment. International Orthopaedics, 41(6), 1071-1072.

Soltani, P., \& Patini, R. (2020). Retracted COVID-19 articles: A side-effect of the hot race to publication. Scientometrics, 125(1), 819-822.

Tennant, J. P. (2018). The state of the art in peer review. FEMS Microbiology letters, 365(19), fny204.

Vervaart, P. (2014). Ethics in online publications. EJIFCC, 25(3), 244.

Walker, R., \& Rocha da Silva, P. (2015). Emerging trends in peer review-a survey. Frontiers in neuroscience, 9, 169.

Wei, Su. J., Rui, Wu. W., Jing Lang, G., Zhao, H., \& Fang Sheng, J. (2020). Erratum to: Transmission risk of patients with COVID-19 meeting discharge criteria should be interpreted with caution. Journal of Zhejiang University: Science B., 21(5), 408-410. https://doi.org/10.1631/jzus.B2000117.

Zhang, L., Zhao, W., Sun, B., Huang, Y., \& Glänzel, W. (2020). How scientific research reacts to international public health emergencies: a global analysis of response patterns. Scientometrics, 124(1), 747-773. 